\title{
A CHARACTERIZATION OF THE ELEMENTS OF THE SOCLE OF A JORDAN ALGEBRA
}

\author{
ANTONIO FERNANDEZ LOPEZ AND EULALIA GARCIA RUS
}

(Communicated by Maurice Auslander)

\begin{abstract}
Let $J$ be a nondegenerate Jordan algebra over a field $K$ of characteristic not 2 . Here we prove that an element $b \in J$ is in the socle if and only if $J$ satisfies dcc on all principal inner ideals $U_{y} J, y \in K b+U_{b} J$. By using this result we show that the socle of a quadratic extension $J_{F}$ of $J$ coincides with the quadratic extension $\operatorname{Soc}(J)_{F}$ of its socle.
\end{abstract}

Throughout this paper $J$ denotes a (linear) Jordan algebra over a field $K$ of characteristic $\neq 2$. Our standard references for Jordan algebras are [6], [7], [11], For $x, y \in J$ we write their product by $x \cdot y$. For $x, y, z \in J$ we write

(1) $L_{x}: J \rightarrow J L_{x} y=x \cdot y$

(2) $U_{x}: J \rightarrow J \quad U_{x} y=2 L_{x}^{2}-L_{x^{2}}$

(3) $\{x y z\}=\left(U_{x+z}-U_{x}-U_{z}\right) y$

(4) $B_{x, y} z=z-\{x y z\}+U_{x} U_{y} z$.

The Jordan algebra $J$ is said to be nondegenerate if $U_{x}=0$ implies $x=0$. An inner ideal is a subspace $I$ of $J$ such that $U_{I} J \subset I$. For any $x, y$ in $J$ we have the principal inner ideal $U_{x} J$, the inner ideal $I(x)=K x+U_{x} J$ generated by $x$, and the Bergmann inner ideal $B_{x, y} J$ [7]. For nondegenerate $J$, the socle $\operatorname{Soc}(J)$ is defined to be the linear span of all minimal inner ideals of $J$; $\operatorname{Soc}(J)=0$ if $J$ does not contain any minimal inner ideal. By [10], if $J$ contains minimal inner ideal then $\operatorname{Soc}(J)$ is a direct sum of simple ideals each of which contains a completely primitive idempotent $e\left(U_{e} J\right.$ is a division Jordan algebra).

An associative algebra $A$ is semiprime iff the Jordan algebra $A^{+}$defined by the product $x \cdot y=\frac{1}{2}(x y+y x)$ is nondegenerate. For semiprime $A$, the (associative) socle of $A$ coincides with the socle of the Jordan algebra $A^{+}$(see [3]). It is well known that an element $a \in A$ is in the socle iff $A$ satisfies dcc on all principal left ideals contained in $A a$. In fact, $A$ satisfies dcc on all left ideals contained in $A a$ for every $a \in \operatorname{Soc}(A)$. In the workshop on Jordan structures held at the University of Ottawa in 1986, McCrimmon settled the

Received by the editors February 21, 1989 and, in revised form, April 19, 1989.

1980 Mathematics Subject Classification (1985 Revision). Primary 17C10, 17C65, 16 A34. 
following Jordan characterization of the elements in the socle of a semiprime associative algebra $A: x \in \operatorname{Soc}(A)$ if and only if $A$ satisfies dcc on all inner ideals contained in $K x+x A x=K x+U_{x} A^{+}$.

This characterization is not true for a general Jordan algebra: the Jordan algebra $J$ of a nondegenerate symmetric bilinear form on a vector space containing an infinite-dimensional totally isotropic subspace satisfies the dcc on principal inner ideals but does not satisfy the dcc on all inner ideals of $U_{1} J=J[8$, p. 465], yet 1 lies in $\operatorname{Soc}(J)=J$. Nevertheless we do obtain an analogous result if we restrict to the principal dcc.

Theorem 1. Let $J$ be a nondegenerate Jordan algebra. An element $b \in J$ is in the socle iff $J$ satisfies dcc on all principal inner ideals $U_{y} J, y \in I(b)$. In particular, every $b \in J$ such that $U_{b} J$ is finite-dimensional belongs to the socle. This last result was proved in [5].

Proof. Suppose first that $b \in \operatorname{Soc}(J)$. By the structure theorem of the socle, we can reduce the problem to the case that $J$ is a simple Jordan algebra with minimal inner ideals. By Litoff theorem for Jordan algebras [1] we have one of the following possibilities:

(i) $b$ belongs to an inner ideal isomorphic to $M_{n}(D)^{+}$for some natural $n$, where $D$ is a division associative algebra.

(ii) $b$ belongs to an inner ideal isomorphic to the Jordan algebra of symmetric elements of $M_{n}(D)^{+}$with respect to an involution $*: M_{n}(D) \rightarrow M_{n}(D)$.

(iii) $J$ is isomorphic to the Jordan algebra of a nondegenerate symmetric bilinear form.

(iv) $J$ is isomorphic to the simple exceptional Jordan algebra 27-dimensional over its center.

In any case $J$ satisfies dcc on all principal inner ideals $U_{y} J, y \in I(b)$ by [8].

Conversely, suppose that $J$ satisfies dcc on all principal inner ideals $U_{y} J$, $y \in I(b)$. Set $\mathfrak{B}=\left\{U_{c} J: c \in I(b), c-b \in \operatorname{Soc}(J)\right\}$. Since $U_{b} J$ belongs to $\mathfrak{B}$, we can choose $U_{c} J$ minimal in $\mathfrak{B}$. If $U_{c} J=0$ then $c=0$ by nondegeneracy of $J$, and hence $b \in \operatorname{Soc}(J)$. Suppose to the contrary that $U_{c} J \neq 0$. Then $U_{c} J$ contains a minimal inner ideal $I$ of the form $I=U_{x} J$ where $x=U_{x} y$ for some $y$ in $J$ [7, p. 106]. For $d=B_{x, y} c$ we have $U_{d} J \subset U_{c} J$, but this inclusion is strict since $x$ lies in $U_{c} J$ but not in $U_{d} J$ ( $B_{x, y} x=0$ but $B_{x, y}$ is the identity on $U_{d} J, B_{x, y} U_{d}=B_{x, y} U\left(B_{x, y} c\right)=B_{x, y} B_{x, y} U_{c} B_{y, x}=B_{x, y} U_{c} B_{y, x}=U_{d}$ since $\left(B_{x, y}\right)^{2}=B_{x, y}$ by [7,(JP25), p. 21]), yet $U_{d} J$ is in $\mathfrak{B}$ since $d$ is in $I(c) \subset I(b)$ and $x$ is in $I \subset \operatorname{Soc}(J)$ so that $d \equiv c \equiv b \bmod \operatorname{Soc}(J)$. This contradicts the minimality of $U_{c} J$ in $\mathfrak{B}$.

The following result answers in the affirmative a question proposed in [2].

Theorem 2. Let $J$ be a nondegenerate Jordan algebra and let $F$ be a quadratic extension of the field $K$. Then the scalar extension $J_{F}=F \otimes_{K} J$ is also nondegenerate with $\operatorname{Soc}\left(J_{F}\right)=\operatorname{Soc}(J)_{F}$. 
Proof. Without loss in generality we may assume that $F=K(\alpha)$ with $\alpha^{2} \in K$ but $\alpha \in F \backslash K$. Then the mapping $a+\alpha b \rightarrow a-\alpha b$ is an involution of the Jordan algebra $J_{F}=J \oplus \alpha J$ over $K$. Hence if $a+\alpha b$ is an absolute zero divisor (a.z.d.) then $a-\alpha b$ is also an a.z.d. By [11, p. 335], $U_{2 a} x=U_{(a+\alpha b)+(a-\alpha b)} x$ is an a.z.d. for all $x \in J$, so $a=0$ by nondegeneracy of $J$. Then $\alpha b$ is an a.z.d. and hence $b=0$ by nondegeneracy of $J$ again. This proves that $J_{F}$ is nondegenerate. Let $e$ be a completely primitive idempotent in $J$ and let $M(e)$ denote the simple ideal of $J$ generated by $e$. By [4, Lemma 2.ii], $e$ belongs to $\operatorname{Soc}\left(J_{F}\right)$ and hence $M(e) \subset \operatorname{Soc}\left(J_{F}\right)$. Since $\operatorname{Soc}(J)$ is the sum of all $M(e), \operatorname{Soc}(J)_{F} \subset \operatorname{Soc}\left(J_{F}\right)$. Conversely, let $x+\alpha y \in \operatorname{Soc}\left(J_{F}\right)$. Since $a+\alpha b \rightarrow a-\alpha b$ is a $K$-involution on $J_{F}$, and the socle is invariant under all ring automorphisms, $x, y \in \operatorname{Soc}\left(J_{F}\right)$. We must show that $x, y \in \operatorname{Soc}(J)$. Suppose to the contrary that $x \notin \operatorname{Soc}(J)$. By Theorem 1 there exists an infinite sequence $\left\{x_{n}\right\} \subset I(x)$ such that the sequence of principal inner ideals $\left\{U\left(x_{n}\right) J\right\}$ is strictly descending. Hence $\left\{U\left(x_{n}\right) J_{F}\right\}$ has the same property, which is a contradiction, by Theorem 1 again, because $x \in \operatorname{Soc}\left(J_{F}\right)$.

\section{ACKNOWLEDGMENT}

The first author would like to thank Professor McCrimmon for his interesting suggestions in order to improve a first proof of Theorem 1.

\section{REFERENCES}

1. P. N. Anh, Simple Jordan algebras with minimal inner ideals, Comm. Algebra 14 (1986), 489-492.

2. M. Benslimane, Charactérisations des algébres de Jordan Banach non commutatives de capacité finie et caractérisation spectrale des algèbres de Jordan Banach non commutatives modulaires annihilatrices, Thesis, Université de Rabat, 1987.

3. A. Fernández López, Modular annihilator Jordan algebras, Comm. Algebra 13 (1985), 25972613.

4. A. Fernández López and A. Rodriguez Palacios, Primitive noncommutative Jordan algebras with nonzero socle, Proc. Amer. Math. Soc. 96 (1986), 199-206.

5. _ On the socle of a noncommutative Jordan algebra, Manuscripta Math. 56 (1986), 269-

6. N. Jacobson, Structure and representations of Jordan algebras, Amer. Math. Soc. Coll. Publ. 39, Providence, Rhode Island, 1968.

7. O. Loos, Jordan pairs, Springer Lecture Notes, Vol. 460, Heidelberg, 1975.

8. K. McCrimmon, Inner ideals in quadratic Jordan algebras, Trans. Amer. Math. Soc. 159 (1971), 445-468.

9. K. Meyberg, Lectures on algebras and triple systems, Lecture Notes, University of Virginia, Charlottesville, Virginia, 1972.

10. J. M. Osborn and M. L. Racine, Jordan rings with nonzero socle, Trans. Amer. Math. Soc. 251 (1979), 375-387.

11. K. A. Zhevlakov, A. M. Slin'ko, I. P. Shestakov and A. I. Shirshov, Rings that are nearly associative, Academic Press, New York, 1982.

Universidad de Malaga, Facultad de Ciencias, Departamento de Algebra, Geometria y Topologia, 29071, Malaga, Spain 